\title{
Serum Cadmium Level Is Positively Associated with Unruptured Intracranial Aneurysm Incidence
}

\author{
Bich Nae Ri Yoon', Jun Beom Lee, ${ }^{2 *}$, Ga Heon Jin, Won Yong Kim \\ 'Department of Family Medicine, Hongik Hospital, Seoul, Korea \\ ${ }^{2}$ Department of Neurology, Hongik Hospital, Seoul, Korea \\ ${ }^{3}$ The Faculty of Beauty Health Sciences, Major in Ophthalmic Optics, Shinhan University, Uijeongbu, Korea
}

Background: Cadmium is a toxic element in cigarette smoke associated with ischemic vascular disease. Its association with cerebral aneurysm is unknown.

Methods: We retrospectively analyzed the medical records of patients with headache who underwent imaging studies between March 2014 and August 2016. An unruptured intracranial aneurysm (UIA) was confirmed by brain magnetic resonance angiography or computed tomography angiography. A control group included age- and sexmatched patients without an UIA. Whole blood and random urine tests were used for detection of cadmium and arsenic levels, respectively. Student t-test was used to compare subject characteristics, mean cadmium and arsenic levels between groups, and differences between groups with small $(<4-\mathrm{mm})$ and large $(\geq 4-\mathrm{mm})$ UIAs. Multivariate regression analysis was used to identify risk factors for aneurysm incidence.

Results: Of 238 patients, 25 had an UIA. Those with an UIA had more pack-years of smoking (19.5 $\pm 3.8 \mathrm{vs.} 12.5 \pm 6.8$, $\mathrm{P}=0.044)$ and higher mean serum cadmium levels ( $1.77 \pm 0.19$ vs. $0.87 \pm 0.21 \mu \mathrm{g} / \mathrm{L}, \mathrm{P}=0.027)$. Arsenic levels showed no difference between groups. (67.4 \pm 23.5 vs. $62.2 \pm 18.3 \mu \mathrm{g} / \mathrm{L}, \mathrm{P}=0.458$ ). There were no significantly different demographic, clinical, or laboratory characteristics between small and large aneurysm groups. According to multivariate analysis, smoking (odds ratio [OR], 1.48; 95\% confidence interval [CI], 1.06-2.33; $\mathrm{P}=0.047$ ) and serum cadmium $>2.0 \mathrm{mcg} / \mathrm{L}(\mathrm{OR}, 1.39 ; 95 \% \mathrm{CI}, 1.15-1.84 ; \mathrm{P}=0.043)$ were associated with aneurysm incidence.

Conclusion: UIA incidence was associated with pack-years of smoking and serum cadmium level, but aneurysm size was not associated with serum cadmium level.

Keywords: Serum; Cadmium; Intracranial Aneurysm; Smoking; Incidence

Received: December 19, 2017, Revised: February 5, 2018, Accepted: March 1, 2018

${ }^{*}$ Corresponding Author: Jun Beom Lee https://orcid.org/0000-0002-7361-3161

Tel: +82-2-2600-0578, Fax: +82-2-2697-4605, E-mail: acupunct@hanmail.net 


\section{INTRODUCTION}

Smoking is a well-known, strong and modifiable risk factor for unruptured intracranial aneurysm (UIA) formation and subarachnoid hemorrhage (SAH) ${ }^{1,2)}$ About $2 \%-3 \%$ of the general population has a UIA. ${ }^{3)}$ According to a previous study, the odds ratio of SAH was 2.4, and 1.7 for UIA in smokers compared to that in the general population. ${ }^{4)} \mathrm{Cad}-$ mium is a toxic element abundant in cigarette smoking, drinking water, and contaminated food, and 2 dominant routes of cadmium absorption are smoke inhalation and diet. ${ }^{5)}$ Although only $1 \%-5 \%$ of ingested, and $25 \%-50 \%$ of inhaled cadmium is absorbed, the biological half-life of cadmium is long due to the low rate of excretion from the body. ${ }^{5,6)}$ Prolonged exposure to cadmium will cause toxic effects in a variety of tissues including the central and peripheral nervous system, kidney, and liver. ${ }^{6}$ Cadmium is known to be an independent risk factor for atherosclerosis and is associated with increased incidence and mortality in coronary and cerebrovascular disease. ${ }^{5,7)}$ However, whether cadmium is associated with UIA incidence specifically has not been fully investigated.

The aim of this study was to determine whether serum cadmium level is positively correlated with UIA incidence and size in comparison with other toxic elements in cigarettes.

\section{METHODS}

\section{Study Population}

Between March 2014 and August 2016, we enrolled 2,127 headache patients who had visited Hongik Hospital neurology outpatient department. Eligible patients had headache, were 18 years or older, never had head trauma or central nervous system infection, underwent brain magnetic resonance angiography (MRA) or computed tomography angiography (CTA), and completed serum cadmium and urine arsenic tests. Those who refused or failed to undergo an imaging study or testing for serum or urine heavy metals were excluded. The patient group was defined as UIA confirmed by brain MRA or brain CTA. The control group was defined as age- and sex-matched patients without UIA. This study corresponds to IRB exemption. And Informed consent was waived.

\section{Data Collection}

All patients and controls were reviewed through medical records. We collected data on demographics (age, sex), smoking status, past history of hypertension, diabetes mellitus, coronary artery disease, peripheral artery occlusive disease, cerebrovascular disease, angiographic findings of aneurysm number, size (or largest aneurysm size if there were multiple aneurysms), location, and type, systolic blood pressure, and hemoglobin Alc (HbAlc), low-density lipoprotein (LDL) cholesterol, serum cadmium, and urine arsenic levels.

\section{Statistical Analysis}

Statistical analyses were performed using PASW SPSS ver. 18.0 Statistical Package Program for Windows (SPSS Inc., Chicago, IL, USA). Patient demographic characteristics and laboratory tests comparing UIApositive and UIA-negative patients were assessed with a paired Student t-test and McNemar's test. Student t-test was used to compare subject characteristics, mean cadmium and arsenic levels, and differences between small $(<4-\mathrm{mm})$ and large $(\geq 4-\mathrm{mm})$ UIAs. Multivariate regression analysis was used to identify risk factors for UIA incidence.

\section{RESULTS}

Of 2,127 enrolled patients, 238 fulfilled brain imaging, serum cadmium, and urine arsenic study requirements. Of these, 25 were UIA-positive and 213 were UIA-negative. Demographic and laboratory profiles of UIA-positive and UIA-negative groups are shown in Table 1. In the UIA-positive group, pack-years of smoking (19.5 \pm 3.8 versus $12.5 \pm 6.8$, $\mathrm{P}=0.044)$ and serum cadmium levels $(1.77 \pm 0.19$ versus $0.87 \pm 0.21 \mu \mathrm{g} / \mathrm{L}$, $\mathrm{P}=0.027$ ) were higher than in the UIA-negative group. Other factors such as age, sex, hypertension, diabetes mellitus, coronary artery disease, peripheral artery occlusive disease, previous stroke, $\mathrm{HbAlc}, \mathrm{LDL}$

Table 1. Baseline patient characteristics

\begin{tabular}{|c|c|c|c|}
\hline Characteristic & UIA positive $(n=25)$ & UIA negative $(n=213)$ & P-value \\
\hline Age (y) & $61.27 \pm 8.47$ & $63.47 \pm 12.8$ & 0.773 \\
\hline Male & $16(64.0)$ & $146(68.5)$ & 0.562 \\
\hline Smoking (pack-years) & $19.5 \pm 3.8$ & $12.5 \pm 6.8$ & 0.044 \\
\hline Hypertension & $17(68.0)$ & $143(67.1)$ & 4.671 \\
\hline Diabetes mellitus & $8(40.0)$ & $92(43.2)$ & 0.867 \\
\hline Coronary artery disease & $3(12.0)$ & $28(13.2)$ & 1.872 \\
\hline Peripheral artery occlusive disease & $2(8.0)$ & $6(2.8)$ & 0.147 \\
\hline Previous stroke & $3(12.0)$ & $23(10.8)$ & 2.198 \\
\hline Hemoglobin A1c (\%) & $7.32 \pm 0.27$ & $7.19 \pm 0.39$ & 1.962 \\
\hline Low-density lipoprotein cholesterol (mg/dL) & $142.3 \pm 11.8$ & $148.2 \pm 30.0$ & 0.302 \\
\hline Serum cadmium ( $\mu \mathrm{g} / \mathrm{L})$ & $1.77 \pm 0.19$ & $0.87 \pm 0.21$ & 0.027 \\
\hline Urine arsenic $(\mu \mathrm{g} / \mathrm{L})$ & $64.1 \pm 17.9$ & $62.2 \pm 18.3$ & 0.458 \\
\hline
\end{tabular}

Values are presented as mean \pm standard deviation for continuous variables or numbers (\%) for categorical variables. UIA, unruptured intracranial aneurysm. 
cholesterol, and urine arsenic level showed no differences between the groups. There were no demographic, clinical, or laboratory differences in characteristics between small $(<4-\mathrm{mm})$ and large $(\geq 4-\mathrm{mm})$ UIA groups (Table 2). According to multivariate logistic regression analysis, smoking (odds ratio [OR], 1.48; 95\% confidence interval [CI], 1.06-2.33) and cadmium level (OR, 1.39; 95\% CI, 1.15-1.84) were associated with increased UIA incidence (Table 3 ).

\section{DISCUSSION}

We observed that UIA incidence was significantly associated with pack-years of smoking and serum cadmium level and succeeded in proving our hypothesis that cadmium but not arsenic was positively associated with UIA. We also found that aneurysm size showed no correlation with serum cadmium level.

Smoking is a well-known modifiable risk factor for SAH and about $40 \%$ of all SAH is attributed to smoking. ${ }^{1)}$ Smoking-associated vascular

Table 2. Characteristics of small $(<4 \mathrm{~mm})$ and large $(\geq 4 \mathrm{~mm})$ UIAS

\begin{tabular}{|c|c|c|c|}
\hline \multirow{2}{*}{ Characteristic } & \multicolumn{2}{|c|}{ UIA size } & \multirow{2}{*}{ P-value } \\
\hline & Small $(<4 \mathrm{~mm})(\mathrm{n}=16)$ & Large $(\geq 4 \mathrm{~mm})(\mathrm{n}=9)$ & \\
\hline Age (y) & $65.8 \pm 10.3$ & $66.2 \pm 9.8$ & 3.729 \\
\hline Male & $9(56.2)$ & $5(55.5)$ & 7.390 \\
\hline Smoking (pack-years) & $22.8 \pm 7.4$ & $21.6 \pm 6.2$ & 5.924 \\
\hline Serum cadmium ( $\mu \mathrm{g} / \mathrm{L})$ & $1.98 \pm 0.42$ & $2.13 \pm 0.20$ & 0.632 \\
\hline Urine arsenic $(\mu \mathrm{g} / \mathrm{L})$ & $78.1 \pm 19.4$ & $82.8 \pm 14.8$ & 1.447 \\
\hline Hypertension & $10(62.5)$ & $6(66.6)$ & 4.671 \\
\hline Diabetes mellitus & $5(31.3)$ & $3(33.3)$ & 0.867 \\
\hline Coronary artery disease & $3(18.7)$ & $2(22.2)$ & 1.872 \\
\hline Peripheral artery occlusive disease & $2(12.5)$ & $1(11.1)$ & 0.147 \\
\hline Previous stroke & $5(31.2)$ & $3(33.3)$ & 2.198 \\
\hline Hemoglobin A1c (\%) & $6.97 \pm 0.12$ & $7.01 \pm 0.39$ & 3.725 \\
\hline Low-density lipoprotein cholesterol (mg/dL) & $144.3 \pm 10.5$ & $146.2 \pm 8.9$ & 4.492 \\
\hline \multicolumn{4}{|l|}{ Location (multiple selection) } \\
\hline Middle cerebral aneurysm bifurcation & $6(37.5)$ & $2(22.2)$ & 2.549 \\
\hline Anterior communicating artery & $7(43.7)$ & $2(22.2)$ & 0.827 \\
\hline Posterior communicating artery & $5(31.3)$ & $4(44.4)$ & 3.192 \\
\hline Other & 0 & $1(11.2)$ & 0.390 \\
\hline \multicolumn{4}{|l|}{ Type (multiple selection) } \\
\hline Saccular & $11(68.7)$ & $5(55.5)$ & 1.592 \\
\hline Fusiform & $7(37.5)$ & $4(44.5)$ & 0.609 \\
\hline
\end{tabular}

Values are presented as mean \pm standard deviation for continuous variables or numbers (\%) for categorical variables.

UIA, unruptured intracranial aneurysm.

Table 3. Independent predictors of unruptured intracranial aneurysm

\begin{tabular}{|c|c|c|c|c|}
\hline \multirow{2}{*}{ Predictors } & \multicolumn{2}{|c|}{ Univariate } & \multicolumn{2}{|c|}{ Multivariate } \\
\hline & $\mathrm{OR}(95 \% \mathrm{Cl})$ & P-value & $\mathrm{OR}(95 \% \mathrm{Cl})$ & P-value \\
\hline Age, per 1-year increase & $1.01(0.99-1.02)$ & 0.245 & $1.02(0.99-1.11)$ & 0.673 \\
\hline Male sex & $1.11(0.94-2.83)$ & 0.068 & $1.82(0.96-3.28)$ & 0.076 \\
\hline Smoking & $1.92(1.12-4.61)$ & 0.038 & $1.48(1.06-2.33)$ & 0.047 \\
\hline Hypertension & $2.37(0.89-3.85)$ & 0.044 & $1.92(1.08-3.43)$ & 0.610 \\
\hline Diabetes mellitus & $1.12(0.79-1.34)$ & 0.537 & $0.85(0.43-1.20)$ & 0.389 \\
\hline Coronary artery disease & $1.32(0.98-1.56)$ & 0.341 & $1.18(0.86-2.34)$ & 0.631 \\
\hline Peripheral artery occlusive disease & $1.01(0.76-1.24)$ & 0.479 & $1.42(0.69-2.95)$ & 0.383 \\
\hline Previous stroke & $1.85(0.45-3.22)$ & 0.097 & $1.73(0.56-2.96)$ & 0.106 \\
\hline \multicolumn{5}{|l|}{ Serum cadmium ( $\mu g / L)$} \\
\hline$<1.0$ & Reference & & Reference & \\
\hline $1.0-2.0$ & $0.97(0.89-1.26)$ & 0.639 & $0.98(0.79-1.33)$ & 0.597 \\
\hline$>2.0$ & $2.09(0.99-4.37)$ & 0.039 & $1.39(1.15-1.84)$ & 0.043 \\
\hline \multicolumn{5}{|l|}{ Urine arsenic ( $\mu \mathrm{g} / \mathrm{L})$} \\
\hline$<50$ & Reference & & Reference & \\
\hline $50-100$ & $0.96(0.77-1.32)$ & 0.472 & $0.95(0.83-1.54)$ & 0.581 \\
\hline$>100$ & $1.02(0.87-1.63)$ & 0.549 & $0.93(0.81-1.12)$ & 0.890 \\
\hline
\end{tabular}

$\mathrm{OR}$, odds ratio; $\mathrm{Cl}$, confidence interval. 
inflammation and arterial oxidative stress are central in the pathogenesis of atherosclerosis and may be responsible for activation of matrix metalloproteinase (MMP). MMPs are known for resorption of extracellular matrix (ECM) components. Excessive breakdown of ECM is a major determinant of aneurysm formation, with loss of the internal elastic lamina, thinning of the media, and ultimately aneurysm formation. ${ }^{2)}$ Ho et al ${ }^{8)}$ also found that smoking had a significant role in aneurysm formation. Our findings are in line with previous studies that smoking is associated with cerebral aneurysm incidence.

Cadmium is ubiquitous and 2 dominant routes of cadmium absorption in humans are smoke inhalation and diet. ${ }^{5)}$ Of more than 5,000 chemical elements and 69 carcinogens in cigarette smoke, cadmium is an independent risk factor for atherosclerosis and increased incidence and mortality of cerebrovascular and coronary artery disease. ${ }^{5,9)}$ The most consistently demonstrated proatherogenic mechanism associated with cadmium is induction of oxidative stress and endothelial dysfunction due to reduced nitric oxide production, increased vascular permeability, inhibited endothelial cell proliferation, upregulated expression of endothelial adhesion molecules, induced apoptosis of endothelial cells, activation of the sympathetic system, inhibition of vasodilator substances, and estrogenic activation. ${ }^{5)}$ Cadmium also affects the central nervous system by means of oxidative stress, impaired neurogenesis, altered gene expression, endocrine disruption, and epigenetic effects. ${ }^{6,10)}$ Chronic exposure to cadmium induces blood-brain barrier (BBB) dysfunction and increases BBB permeability. ${ }^{11)}$

Despite positive associations between cadmium and UIA, however, we found no significant effects of arsenic on aneurysm incidence. Arsenic is a ubiquitous element that is widely distributed in the environment and is mainly transported by water. Humans are exposed to inorganic and organic arsenic through environmental, medicinal, and occupational sources by oral, respiratory, or dermal routes. It is reported that arsenic might affect thrombocytes, which play an important role in cardiovascular diseases. ${ }^{12)}$ Chiou et al. ${ }^{13)}$ stated that cerebrovascular disease may be induced by long-term exposure to inorganic arsenic.

To compare serum cadmium levels, we dichotomized UIA size as $<4$ $\mathrm{mm}$ and $\geq 4 \mathrm{~mm}$. In previous studies, aneurysm size, location, sex, and ethnic background influenced risk stratification for aneurysm rupture. In addition, an aneurysm size ratio (the ratio of aneurysm size to parent artery diameter) $>4$ was reportedly significantly associated with increased risk of aneurysm rupture. ${ }^{14)}$ Korja et al. ${ }^{15)}$ reported that ruptured intracranial aneurysms (RIAs) of the anterior communicating artery were more frequently found in men than in women. In addition, RIAs of the ophthalmic artery had a median size of $11 \mathrm{~mm}$, whereas the median size of RIAs of the pericallosal artery was $6 \mathrm{~mm}$. Of all RIAs, $68 \%$ were $<10 \mathrm{~mm}$ in maximum diameter. ${ }^{15)}$ Jeong et al. ${ }^{16)}$ reported RIA size in anterior, middle, and posterior communicating arteries of 3-6 $\mathrm{mm}, 3-6 \mathrm{~mm}$, and 4-6 mm, respectively, based on a Korean population study. Murayama et al. ${ }^{17)}$ reported that UIAs $>5 \mathrm{~mm}$ were at greater risk of rupture compared with aneurysms measuring 2-4 mm (unadjusted hazard ratio, 12.24 ; 95\% CI, 7.15-20.93) in the Japanese popu- lation. From the above studies, we thought it would be most effective to dichotomize aneurysm size as $<4 \mathrm{~mm}$ and $\geq 4 \mathrm{~mm}$ to represent low and high risk of aneurysm rupture. According to our findings, there was no significant difference in serum cadmium level between small ( $<4 \mathrm{~mm}$ ) and large ( $\geq 4 \mathrm{~mm}$ ) UIAs.

Our study had several limitations. First, this was a retrospective study of all participants with headache. Potential UIA patients who were asymptomatic were excluded, which may have biased the diagnostic rate toward overestimation. Second, sources of cadmium exposure other than smoking such as the workplace or past and current place of residence were not identified. In addition, we could not determine whether cadmium intoxication was acute or chronic because neither organ tissue biopsy nor urine beta-2 microglobulin testing (an indicator of renal dysfunction with chronic cadmium exposure) was performed.

However, our study had several strengths. First, the population in our study was well defined because patients with or without UIA were seen in the same center. Second, using a large number of patients (total=238), we found a statistically significant increased association of UIA with pack-years of smoking and serum cadmium levels. Third, our study suggests that mechanisms other than smoking may be independent risk factors for aneurysm incidence and enlargement.

In summary, the present study supports the hypothesis that cigarette smoking and serum cadmium concentration are associated with increased cerebral aneurysm incidence. These findings suggest that common pathophysiology may be shared between cerebral atherosclerosis and aneurysm formation in the context of smoking and cadmium intoxication from all sources. These results highlight the importance of smoking cessation. A good risk-reduction strategy for UIA and SAH may include routine serum cadmium testing when a UIA is incidentally discovered.

\section{CONFLICT OF INTEREST}

No potential conflict of interest relevant to this article was reported.

\section{ORCID}

Bich Nae Ri Yoon: https://orcid.org/0000-0001-8891-5587

Jun Beom Lee: https://orcid.org/0000-0002-7361-3161

Ga Heon Jin: https://orcid.org/0000-0002-9012-647X

Won Yong Kim: https://orcid.org/0000-0003-2117-5099

\section{REFERENCES}

1. Juvela S, Poussa K, Lehto H, Porras M. Natural history of unruptured intracranial aneurysms: a long-term follow-up study. Stroke 2013;44: 2414-21.

2. Chalouhi N, Ali MS, Starke RM, Jabbour PM, Tjoumakaris SI, Gonzalez LF, et al. Cigarette smoke and inflammation: role in cerebral aneurysm formation and rupture. Mediators Inflamm 2012;2012:271582.

3. Vlak MH, Rinkel GJ, Greebe P, Algra A. Independent risk factors for in- 
tracranial aneurysms and their joint effect: a case-control study. Stroke 2013;44:984-7.

4. Matsumoto K, Akagi K, Abekura M, Ohkawa M, Tasaki O, Oshino S. Cigarette smoking increases the risk of developing a cerebral aneurysm and of subarachnoid hemorrhage. No Shinkei Geka 1999;27:8315.

5. Santos-Gallego CG, Jialal I. Cadmium and atherosclerosis: heavy metal or singing the blues? Atherosclerosis 2016;249:230-2.

6. Wang B, Du Y. Cadmium and its neurotoxic effects. Oxid Med Cell Longev 2013;2013:898034.

7. Peters JL, Perlstein TS, Perry MJ, McNeely E, Weuve J. Cadmium exposure in association with history of stroke and heart failure. Environ Res 2010;110:199-206.

8. Ho AL, Lin N, Frerichs KU, Du R. Smoking and intracranial aneurysm morphology. Neurosurgery 2015;77:59-66.

9. Knoflach M, Messner B, Shen YH, Frotschnig S, Liu G, Pfaller K, et al. Non-toxic cadmium concentrations induce vascular inflammation and promote atherosclerosis. Circ J 2011;75:2491-5.

10. Socha K, Borawska MH. Mineral components in aneurysms. In: Murai Y, editor. Aneurysm [Internet]. London: IntechOpen Limited; 2012 [cited 2017 Oct 2]. Available from: https://www.intechopen.com/ books/aneurysm/mineral-components-in-aneurysms.

11. Park SL, Kim YM, Ahn JH, Lee SH, Baik EJ, Moon CH, et al. Cadmium stimulates the expression of vascular cell adhesion molecule-1 (VCAM-1) via p38 mitogen-activated protein kinase (MAPK) and JNK activation in cerebrovascular endothelial cells. J Pharmacol Sci 2009; 110:405-9.

12. Lee MY, Bae ON, Chung SM, Kang KT, Lee JY, Chung JH. Enhancement of platelet aggregation and thrombus formation by arsenic in drinking water: a contributing factor to cardiovascular disease. Toxicol Appl Pharmacol 2002;179:83-8.

13. Chiou HY, Huang WI, Su CL, Chang SF, Hsu YH, Chen CJ. Dose-response relationship between prevalence of cerebrovascular disease and ingested inorganic arsenic. Stroke 1997;28:1717-23.

14. Rahman M, Smietana J, Hauck E, Hoh B, Hopkins N, Siddiqui A, et al. Size ratio correlates with intracranial aneurysm rupture status: a prospective study. Stroke 2010;41:916-20.

15. Korja M, Kivisaari R, Rezai Jahromi B, Lehto H. Size and location of ruptured intracranial aneurysms: consecutive series of 1993 hospitaladmitted patients. J Neurosurg 2017;127:748-53.

16. Jeong YG, Jung YT, Kim MS, Eun CK, Jang SH. Size and location of ruptured intracranial aneurysms. J Korean Neurosurg Soc 2009;45:11-5.

17. Murayama Y, Takao H, Ishibashi T, Saguchi T, Ebara M, Yuki I, et al. Risk analysis of unruptured intracranial aneurysms: prospective 10year cohort study. Stroke 2016;47:365-71. 